\title{
Kussmaul's sign for the diagnosis of right ventricular myocardial infarction: a systematic review and meta-analysis of diagnostic test accuracy studies
}

\author{
Emily Dubé ${ }^{\prime} \cdot$ Michael Crozier $^{1,2} \cdot$ Allan Middleton $^{1} \cdot$ Brittany Best $^{1} \cdot$ Robert Ohle ${ }^{1,3}$ \\ Received: 4 June 2020 / Accepted: 28 August 2020 / Published online: 4 January 2021 \\ (c) Canadian Association of Emergency Physicians (CAEP)/ Association Canadienne de Médecine d'Urgence (ACMU) 2021
}

\begin{abstract}
Introduction Kussmaul's sign, the absence of a drop in jugular venous pressure or a paradoxical increase in jugular venous pressure on inspiration, can be evaluated as an indicator of right ventricular myocardial infarction. Right ventricular myocardial infarction complicates $30-50 \%$ of inferior myocardial infarctions and is associated with increased mortality when compared to inferior myocardial infarction without right ventricular involvement. Early recognition allows maintenance of preload. We reviewed the diagnostic test accuracy studies for Kussmaul's sign for diagnosis of right ventricular myocardial infarction.

Methods We conducted a librarian-assisted search using PubMed, Medline, Embase, and the Cochrane database from 1965 to October 2019. Only English language restriction was imposed. We identified studies that assessed patients presenting to a hospital with a suspected myocardial infarction who underwent an assessment for Kussmaul's sign and a diagnostic test for right ventricular myocardial infarction. Four independent reviewers extracted data from relevant studies. Study quality was assessed using the QUADAS-2 tool. A bivariate random effects meta-analysis was performed.

Results We identified 122 studies; ten were selected for full review. Eight studies had comparable populations with a total of 469 consecutive patients admitted with acute inferior myocardial infarction and were included in the analysis. Prevalence of right ventricular myocardial infarction was 36\% (confidence interval [CI] 95\% 31.8-40.5). All reference standards were combined. Kussmaul's sign had a sensitivity of $62.5 \%$ (44.6, 77.5), specificity $90 \%(73.0,96.8)$, negative likelihood ratio (LR) $0.2(0.1-0.8)$ and positive LR $5.8(2.5,13.3)$.

Conclusion In the presence of acute myocardial infarction, Kussmaul's sign is specific for acute right ventricular myocardial infarction and may serve as an important clinical sign of right ventricular dysfunction requiring preload preserving management.
\end{abstract}

Keywords Kussmaul's sign · Right ventricular myocardial infarction · Clinical exam · Diagnostic accuracy test

\section{Résumé}

Introduction Le signe de Kussmaul, l'absence de baisse de la pression veineuse jugulaire ou une augmentation paradoxale de la pression veineuse jugulaire à l'inspiration, peut être évalué comme un indicateur d'infarctus du myocarde ventriculaire droit. Elle complique 30 à 50\% des infarctus du myocarde inférieur et est associée à une mortalité accrue par rapport à l'infarctus du myocarde inférieur sans atteinte ventriculaire droite. Une détection précoce permet de maintenir la précharge. Nous avons examiné les études sur la précision des tests de diagnostic du signe de Kussmaul pour le diagnostic de l'infarctus du myocarde ventriculaire droit.

\section{Emily Dubé}

edube@nosm.ca

1 Northern Ontario School of Medicine, 935 Ramsey Lake

Road, Sudbury, ON P3E2C6, Canada

2 Canadian Armed Forces, Ottawa, Canada

3 Health Science North, Sudbury, Canada 
Méthodes Nous avons mené une recherche assistée par un bibliothécaire à l'aide de PubMed, Medline, Embase et la base de données Cochrane de 1965 à octobre 2019. Seule la restriction de la langue anglaise a été imposée. Nous avons identifié des études qui ont évalué des patients se présentant à un hôpital avec un infarctus suspecté de myocarde et qui ont subi une évaluation du signe de Kussmaul et un test de diagnostic pour un infarctus du myocarde ventriculaire droit. Quatre examinateurs indépendants ont extrait les données d'études pertinentes. La qualité de l'étude a été évaluée à l'aide de l'outil QUADAS-2. Une méta-analyse bivariée des effets aléatoires a été réalisée.

Résultats Nous avons recensé 122 études ; dix ont été sélectionnées pour un examen complet. Huit études avaient des populations comparables avec un total de 469 patients consécutifs admis avec un infarctus aigu du myocarde inférieur et ont été inclus dans l'analyse. La prévalence de l'infarctus du myocarde ventriculaire droit était de $36 \%$ (intervalle de confiance [IC] $95 \%$ 31,8-40,5). Toutes les normes de référence ont été combinées. Le signe de Kussmaul avait une sensibilité de 62,5 $\%(44,6,77,5)$, de spécificité $90 \%$ (73,0, 96,8), un rapport de vraisemblance négatif (LR) 0,2 (0,1-0,8) et de LR positif 5,8 $(2,5,13,3)$.

Conclusion En présence d'un infarctus aigu du myocarde, le signe de Kussmaul est spécifique à l'infarctus aigu du myocarde ventriculaire droit et peut constituer un signe clinique important de dysfonctionnement du ventricule droit nécessitant une gestion de la préservation de la précharge.

\section{Clinician's capsule}

What is known about this topic?

Kussmaul's sign has been used as a clinical sign for right ventricular myocardial infarction, but its diagnostic accuracy is unknown.

\section{What did this study ask?}

Can Kussmaul's sign, a paradoxical rise or absence of a drop in jugular venous pressure, aid in the diagnosis of right ventricular myocardial infarction?

\section{What did this study find?}

Kussmaul's sign is an excellent positive predictor of right ventricular myocardial infarction likely rivaling ECG's V4R lead in diagnostic accuracy.

\section{Why does this study matter to clinicians?}

In patients with suspected inferior myocardial infarction and a positive Kussmaul's sign, manage their preload and hold the nitro.

\section{Introduction}

The clinical and haemodynamic features of right ventricular myocardial infarction were first described by Cohn et al. [1], leading to its recognition as a distinct clinical entity. Right ventricular myocardial infarction occurs when there is occlusion of the coronary arteries supplying the right side of the heart [2]. The incidence of isolated right ventricular myocardial infarction is rare, accounting for merely $2 \%$ of autopsies [2]. Despite its rarity, right ventricular myocardial infarction complicates $30-50 \%$ of inferior myocardial infarction and $10 \%$ anterior myocardial infarction [2-4].
This condition poses unique diagnostic and management challenges and is often associated with higher in-hospital morbidity $[2,5]$. The mortality of right ventricular myocardial infarction is $25 \%$ higher than that of an inferior myocardial infarction not associated with right ventricular involvement $[2,3]$. When an right ventricular myocardial infarction is suspected, it is necessary to maintain preload pressure by treating with fluids and vasopressors as well as avoiding the use of nitroglycerin, diuretic and narcotic medication $[3,6]$.

Right ventricular myocardial infarction presents with typical manifestations of myocardial infarction such as chest pain, nausea, vomiting and diaphoresis $[3,6]$. It is often accompanied by a haemodynamic triad of hypotension, elevated jugular venous pressure and clear lung fields $[2-4,6]$. The correct and early diagnosis of right ventricular myocardial infarction improves clinical outcomes, decreases electrical and mechanical complications and improves overall short-term and long-term prognoses [3, 4]. Thus, clinicians must hold a high index of suspicion for right ventricular myocardial infarction when managing a patient with undifferentiated chest pain. In current clinical practice, ST-segment elevation $\geq 1.0 \mathrm{~mm}$ in the right sided precordial electrocardiogram (ECG) V4R lead is used to aid in diagnosis of right ventricular myocardial infarction [7]. This finding is estimated to be $70-100 \%$ sensitive and $87-100 \%$ specific $[2,7,8]$. However, these changes are transient in nature and may be absent in up to half of patients with right ventricular myocardial infarction ten hours after the onset of chest pain [9].

Decreased right ventricular filling can result in the absence of a drop in jugular venous pressure or a paradoxical increase in jugular venous pressure on inspiration known as Kussmaul's sign [10-12]. Kussmaul's sign is observed in all conditions that restrict right ventricular filling. The differential diagnosis for a positive Kussmaul's 
sign includes constrictive pericarditis, right-sided heart failure, pulmonary hypertension, right ventricular myocardial infarction, tricuspid stenosis and massive pulmonary embolism [10-12]. A positive Kussmaul's sign may be a useful clinical indicator of right ventricular myocardial infarction while the patient's presentation remains undifferentiated. Currently, there is no gold standard for the diagnosis of right ventricular myocardial infarction, although autopsy, coronary angiography and ventricular scintigraphy have been proposed $[5,13]$. The following reference standards have been used: echocardiography, ECG with right sided precordial leads and haemodynamic studies.

The primary purpose of this study was to perform a rigorous systematic review and meta-analysis of diagnostic test accuracy studies to assess the sensitivity and specificity of Kussmaul's sign for diagnosis of right ventricular myocardial infarction in acute myocardial infarction.

\section{Materials and methods}

\section{Data ssources and search strategy}

We performed a librarian-assisted systematic search of PUBMED, EMBASE (1965-October 2019), MEDLINE (Ovid) (1965-October 2019) and the Cochrane Database. The search utilized the Boolean strategy with "Kussmaul* and Sign" as search indicators. References from the final collection of full-text articles were investigated to identify any further studies. With "English language" being considered the only restriction, four reviewers independently conducted the search. Search results were reported according to PRISMA guidelines for diagnostic test accuracy studies (Fig. 1).

\section{Study selection and review procedure}

A small number of studies applicable to Kussmaul's sign in right ventricular myocardial infarction have been published. We included those that met the following criteria: (1) population-adult patients with chest pain presenting to a hospital; (2) test-Kussmaul's sign assessed by clinical exam; (3) reference standard-ECG, echocardiogram and haemodynamic studies; and (4) outcome—right ventricular myocardial infarction. This criterion was defined a priori.

Four reviewers independently assessed all included studies from title and abstracts against the inclusion criteria. Studies that met the inclusion criteria underwent a full text evaluation by the four reviewers, including a methodological and bias assessment performed using the standard QUADAS-2 tool for primary diagnostic accuracy studies [14]. Disagreements between reviewer article selection, bias assessment, and data extraction were resolved through discussion.

\section{Individual evidence quality appraisal}

The QUADAS-2 tool for systematic reviews to evaluate the quality of evidence was applied by four independent reviewers. They used a priori criteria separated by four domains to evaluate a study's risk of bias and degree of applicability.

Domain 1: patient selection

- Risk of bias: For each study selected, the inclusion and exclusion criteria were evaluated to determine whether patients were presenting with undifferentiated chest pain prior to enrollment in the study. If patients were selected after receiving a diagnosis, the risk of bias was recorded as high. If studies did not report inclusion or exclusion criteria, the risk of bias was reported as unclear. If the patients were selected consecutively or randomly and appropriate inclusion and exclusion criteria were reported, the study was recorded as low risk of bias.

- Applicability concerns: If patients had a diagnosis of right ventricular myocardial infarction prior to the clinical assessment of Kussmaul's sign, the applicability concerns were recorded as high.

Domain 2: index tests

- Risk of bias: If the method of assessment of Kussmaul's sign was not described, the bias of the index test was assessed as unclear. If, however, the study described the method of assessment of jugular venous pressure but not Kussmaul's sign, the risk of bias in the index test was marked as low.

- Applicability concerns: If non-emergency physicians assessed the patient for Kussmaul's sign, applicability concern of the index was marked as high.

Domain 3: reference standard

- Risk of bias: If the patient did not undergo an appropriate reference standard such as echocardiography, ECG, computed tomography (CT) angiography or nuclear medicine scan, the risk of bias was marked as high. If the reference standard result was interpreted with knowledge of the presence or absence of Kussmaul's sign, the risk of bias was recorded as high.

- Applicability concerns: If the study's target condition resulted in right ventricular dysfunction, but was not accompanied by chest pain, the concerns regarding applicability were marked as high. 


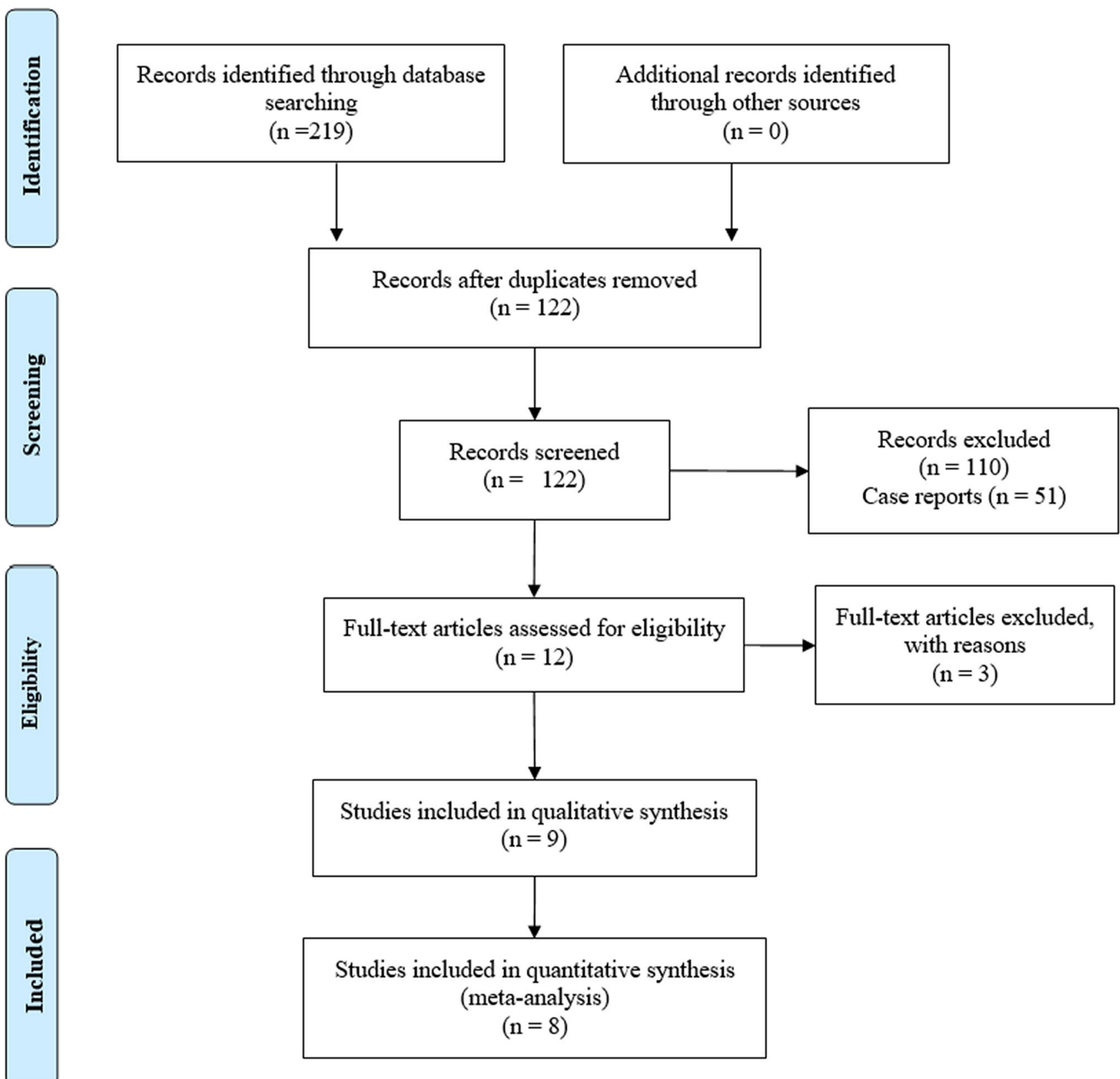

Fig. 1 PRISMA flow diagram

Domain 4: flow and timing

- Risk of bias: If the assessment of right ventricular myocardial infarction by the reference standard was delayed, misclassification may have occurred. If the reference standard was not performed on the same day as the clinical assessment of Kussmaul's sign risk of bias was marked as high. If assessment of Kussmaul's sign was not completed on initial presentation of chest pain, but after initiation of management, the risk of bias was marked as high.

\section{Diagnostic accuracy_data extraction and statistical analysis}

The diagnostic accuracy of Kussmaul's sign was determined through data extraction to populate a $2 \times 2$ table comparing Kussmaul's sign to the diagnosis of right ventricular myocardial infarction by a reference standard (Table 1). Statistical analyses were performed using the Metafor package in $\mathrm{R}$ statistical software, version 3.5.3 [15]. In the case of a cell count with zero, a fixed value of 0.5 was added to mitigate 
Table $12 \times 2$ table for included studies

\begin{tabular}{lccccc}
\hline Study & Total $(n)$ & True positives & False positives & True negatives & $\begin{array}{c}\text { False } \\
\text { nega- } \\
\text { tives }\end{array}$ \\
\hline Pandey & 37 & 7 & 3 & 22 & 5 \\
Cintron & 44 & 16 & 14 & 14 & 0 \\
Dell Italia & 53 & 8 & 0 & 45 & 0 \\
Baigrie & 35 & 17 & 4 & 4 & 10 \\
Sinha & 50 & 9 & 0 & 30 & 11 \\
Sewdardsen & 90 & 21 & 3 & 61 & 5 \\
Hashmat & 110 & 11 & 0 & 71 & 28 \\
Bellamy & 50 & 12 & 4 & 25 & 9 \\
\hline
\end{tabular}

computational problems [15]. To compute diagnostic sensitivity, specificity and likelihood ratios, a bivariate randomeffects model was used for the three combined reference standards. Heterogeneity was visually assessed with forest plots. Subset analysis for the three reference standards was decided on a priori and completed.

\section{Results}

\section{Characteristics of included studies}

Our search identified 122 studies for abstract and title review (Fig. 1). Ten were selected for full text review; their detailed characteristics are presented in Table 2. Eight of these ten studies were included in the statistical analysis [2, 9, 16-21]. The two excluded studies did not have comparable study designs [22, 23].

The risk of bias summary is shown in Fig. 2. All eight of the included studies were prospective, observational studies representing a total of 469 consecutive patients admitted to the coronary care unit with inferior myocardial infarction. Patients were assessed for Kussmaul's sign on clinical exams and underwent a reference test (ECG, echocardiogram, nuclear medicine, or haemodynamic studies) to determine right ventricular involvement within $72 \mathrm{~h}$ of their initial assessment. The prevalence of right ventricular myocardial infarction was 36\% (CI 95\% 31.8-40.5).

\section{Quality assessment}

Figure 2 presents the results of the quality assessment, deeming the overall condition of the included studies to be acceptable. For all included studies, patient selection was rated as problematic for its risk of both bias and applicability since patients were selected with a known inferior myocardial infarction. The reference standard and flow and timing were also noted as common areas of weakness for risk of bias. They were typically interpreted with knowledge of the index test and the timing of the reference test was often unclear or not completed on the same day as the index test. The index test had low risk of bias and unclear or high risk of applicability concerns since the assessors of Kussmaul's sign were either unknown or non-emergency physicians.

\section{Reference standard and accuracy of Kussmaul's sign}

Reference standards for the diagnosis of right ventricular myocardial infarction included echocardiography $(n=3)$, ECG with right-sided precordial leads $(n=3)$, nuclear medicine scan $(n=1)$ and haemodynamic studies $(n=3)$. A single gold standard for the diagnosis of right ventricular myocardial infarction is lacking, thus all reference standards were combined. Kussmaul's sign had a sensitivity of $62.5 \%$ (CI 95\% 44.6, 77.5), specificity of 90\% (CI 95\% 73.0, 96.8), negative LR of 0.2 (CI 95\% 0.1-0.8) and positive LR of 5.8 (CI 95\% 2.5, 13.3) (Fig. 3). Subset analysis of echocardiography, ECG and haemodynamic studies revealed sensitivity of $61.5 \%, 80.2 \%$ and $75.4 \%$, respectively and specificity of $88.0 \%, 86.1 \%, 91.1 \%$.

\section{Heterogeneity}

Clinical heterogeneity: Patients were similarly recruited and selected among studies, indicating a comparable prevalence of right ventricular myocardial infarction among most studies (Table 2). In contrast, the Baigrie study had an unusually high prevalence of right ventricular myocardial infarction. For this reason, a sensitivity analysis was performed. Its exclusion did not appreciably alter the statistical results. Four proxies were used for the classification of right ventricular myocardial infarction (ECG, echocardiogram, nuclear medicine scan and haemodynamic studies), which introduced a high level of clinical heterogeneity (Table 2).

Statistical heterogeneity: Statistical heterogeneity was assessed visually by forest plots. No pattern was noted between reference standards. A high level of heterogeneity 


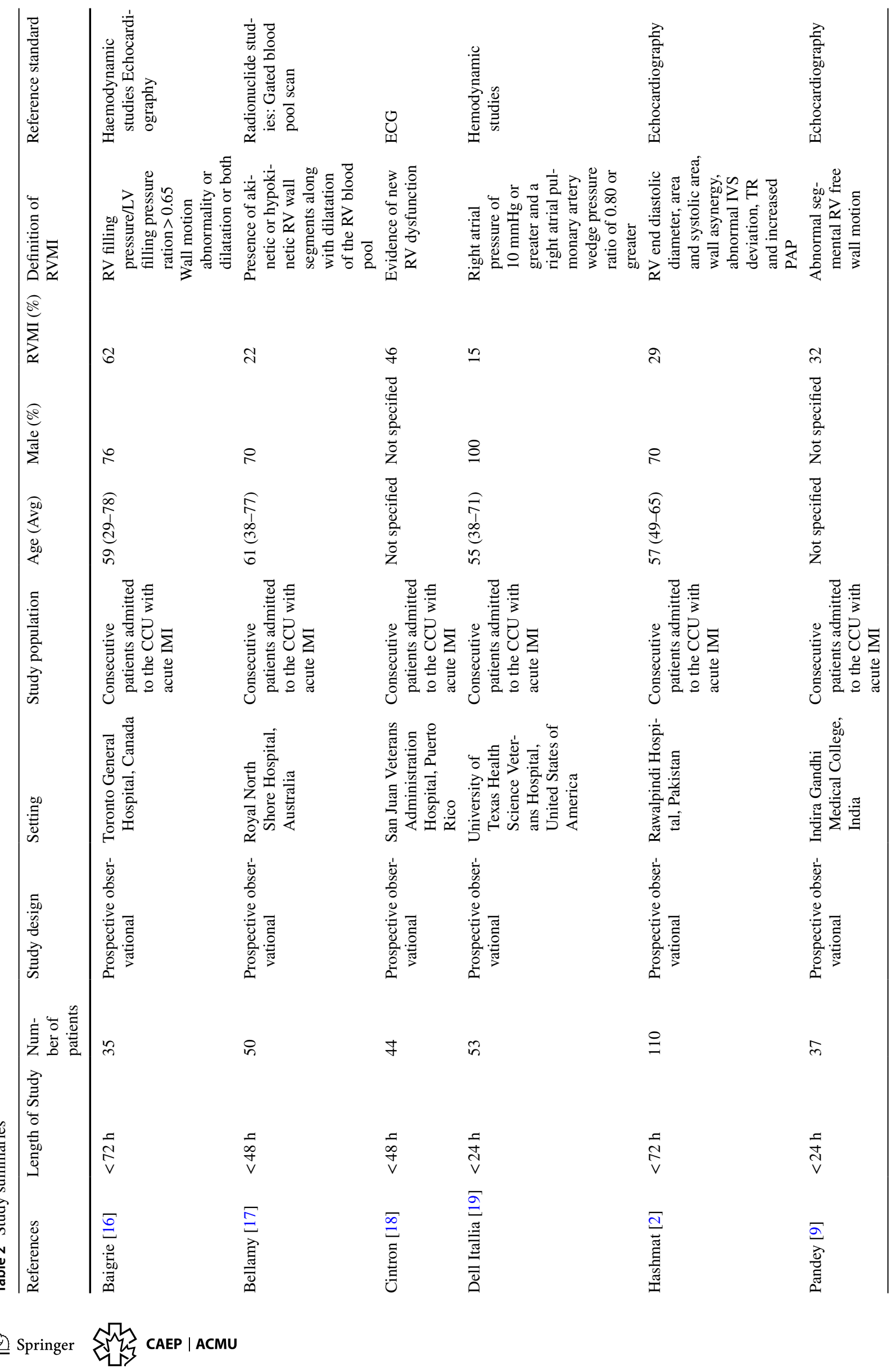




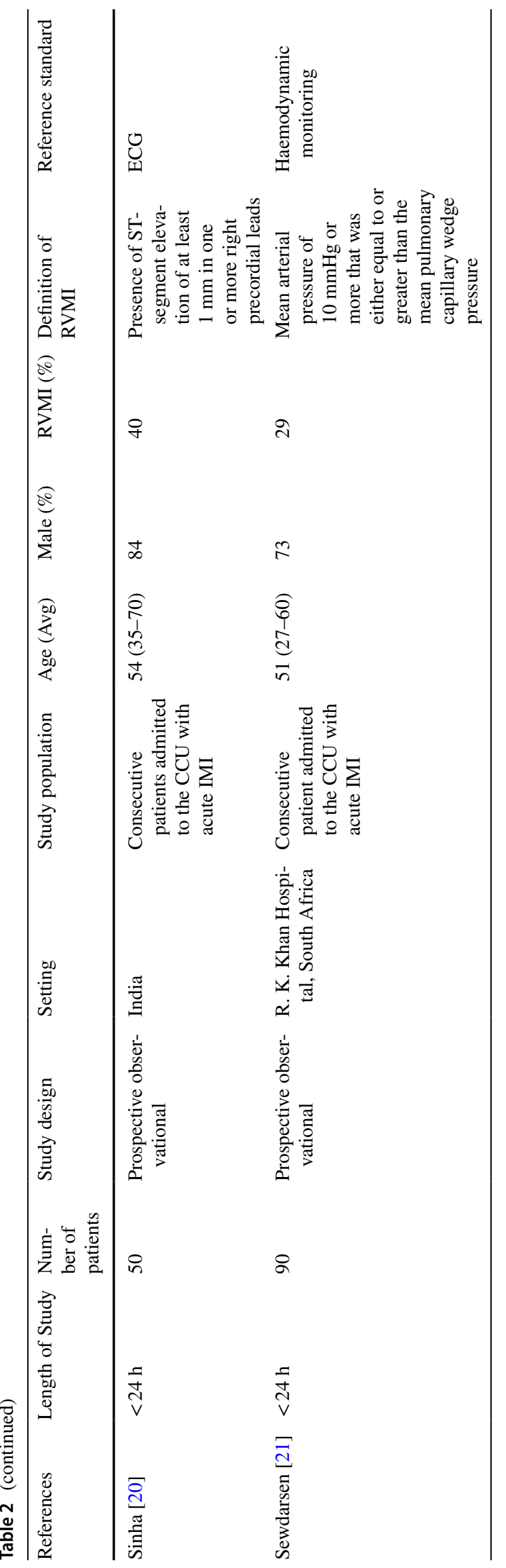

was expected in this meta-analysis of diagnostic test accuracy studies.

\section{Discussion}

\section{Principle findings}

This meta-analysis indicates that a positive Kussmaul's sign is a useful clinical indicator of right ventricular myocardial infarction in patients with inferior myocardial infarction. Kussmaul's sign is a known clinical sign of elevated right ventricular pressures, and thus an indicator of right ventricular dysfunction. Approximately 50 case reports describe its usefulness in the identification of various clinical conditions such as right ventricular right ventricular myocardial infarction myocardial infarction, pulmonary embolism, and constrictive pericarditis (Fig. 1) [6, 24-26]. Despite this, Kussmaul's sign has not been studied robustly. We identified eight studies representing a total of 469 consecutive patients admitted to a coronary care unit with inferior myocardial infarction. These studies demonstrate that Kussmaul's sign has an excellent positive LR of 5.8 (CI 95\% 2.5, 13.3) for right ventricular myocardial infarction with a specificity of 90\% (CI 95\% 73.0, 96.8), sensitivity of 62.5\% (CI 95\% 44.6, 77.5) and negative LR of 0.2 (CI 95\% 0.1-0.8).

Among seven of the eight studies, prevalence of right ventricular myocardial infarction was within the expected range $[2,6]$. The Baigrie study had a prevalence of right ventricular myocardial infarction of $62 \%$. In contrast to the other studies, the Baigrie study attempted to identify the range of right ventricular involvement in inferior myocardial infarction. Given this objective, the definitions used to classify patients was ambiguous. Furthermore, $40 \%$ of the sample was excluded due to inability to assess the patients' neck veins. Its exclusion did not result in a significant change in our statistical analysis. The eight studies included were dated with six of the eight being published prior to 1990 . The remaining two were published in 2006 and 2010.

All included studies had clinically significant heterogeneity due to the different reference standards and definition of right ventricular myocardial infarction used. Subset analysis showed that the reference standards were similar in specificity and had variable sensitivity. Recorded haemodynamic and ECG studies had the highest sensitivity, while echocardiography had the lowest. Three of the eight studies directly compared their reference standard to both Kussmaul's sign and ECG findings. Bellamy et al. [17] found that compared to nuclear medicine scan, ECG was 50\% sensitive and $71 \%$ specific for right ventricular myocardial infarction while Kussmaul's sign was $59 \%$ sensitive and $86 \%$ specific. Sewdarsen et al. [21] found ECG findings to be $96 \%$ sensitive and 
Fig. 2 Summary of QUADAS-2 assessment of included studies

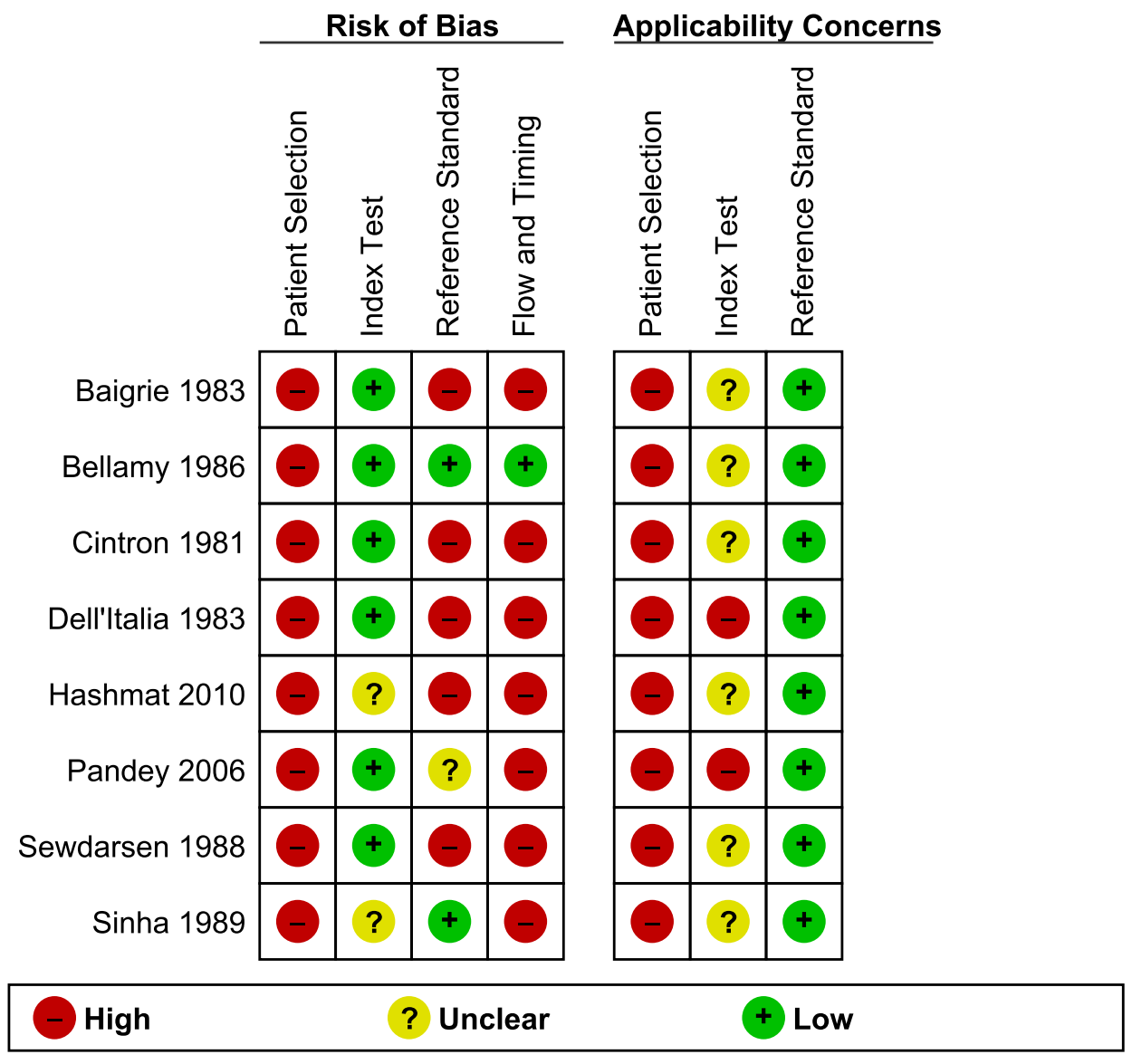

$77 \%$ specific, while Kussmaul's sign was $81 \%$ sensitive and $95 \%$ specific using haemodynamic monitoring as a reference standard. Lastly, Pandey et al. [9] used echocardiography as a reference standard and identified ECG to be $67 \%$ sensitive, and $88 \%$ specific, and Kussmaul's sign to be $58 \%$ sensitive and $88 \%$ specific. The reference standards measure haemodynamic function, electrical conductivity changes and myocardial motility and anatomical changes, respectively, as proxies for right ventricular myocardial infarction. The significance of these statistical differences for each of the reference standard is best interpreted within the clinical context; Kussmaul's sign remains a clinical sign that discerns clinically important changes in right ventricular function and may rival the V4R ECG lead for diagnosis of right ventricular myocardial infarction in the setting of acute myocardial infarction.

\section{Limitations}

Despite representing a large study population with similar study design and inclusion criteria, the eight included studies had several limitations. To begin, these studies were dated with limited descriptions of the study population and were not designed to determine the clinical usefulness of Kussmaul's sign in diagnosis of undifferentiated chest pain. Thus, the studies limited the inclusion criteria to inferior myocardial infarction and examined its complication by right ventricular myocardial infarction. Despite representing a limitation, this approach remained a useful framework within which to conduct a meta-analysis. The assessment of Kussmaul's sign was conducted by nonemergency physicians, typically cardiologists, which limits the applicability of our results in the target clinical environment. Furthermore, the reference standard was usually interpreted with the knowledge of the index test, thus introducing notable bias. Lastly, the clinical and statistical heterogeneity remains a limitation.

\section{Clinical implications and future research}

Our assessment of the literature indicates that Kussmaul's sign is an excellent positive predictor of right ventricular myocardial infarction in the context of inferior myocardial 


\begin{tabular}{|c|c|c|c|}
\hline \multicolumn{4}{|c|}{ Sensitivity } \\
\hline Studies & Estimate $(95 \%$ & C.I.) & $\mathrm{TP} /(\mathrm{TP}+\mathrm{FN})$ \\
\hline Pandey 1 & $0.583(0.308,0$ & $0.815)$ & $7 / 12$ \\
\hline Cintron 2 & $0.971(0.664,0$ & $0.998)$ & $16 / 16$ \\
\hline Dell Italia 3 & $0.944 \quad(0.495,0$ & $0.997)$ & $8 / 8$ \\
\hline Baigrie 4 & $0.630(0.438,0$ & $0.788)$ & $17 / 27$ \\
\hline Sinha 5 & $0.452(0.259,0$ & $0.661)$ & $9 / 20$ \\
\hline Sewdardsen 6 & $0.808 \quad(0.613,0$ & $0.918)$ & $21 / 26$ \\
\hline Hashmat 7 & $0.287(0.169,0$ & $0.445)$ & $11 / 39$ \\
\hline Bellamy 8 & $0.571(0.360,0$ & $0.760)$ & $12 / 21$ \\
\hline Overall $\left(I^{\wedge} 2=7342 \%, P<0.001\right)$ & $0.625(0.446,0$ & $0.775)$ & $101 / 169$ \\
\hline \multicolumn{4}{|c|}{ Specificity } \\
\hline Studies & Estimate $(95 \%$ & C.I.) & $\mathrm{TN} /(\mathrm{FP}+\mathrm{TN})$ \\
\hline Pandey 1 & $0.880 \quad(0.687$, & $0.961)$ & $22 / 25$ \\
\hline Cintron 2 & $0.500 \quad(0.326$, & $0.674)$ & $14 / 28$ \\
\hline Dell Italia 3 & $0.989(0.849$, & $0.999)$ & $45 / 45$ \\
\hline Baigrie 4 & $0.500 \quad(0.200$ & $0.800)$ & $4 / 8$ \\
\hline Sinha 5 & $0.984 \quad(0.789$, & $0.999)$ & $30 / 30$ \\
\hline Sewdardsen 6 & $0.953(0.865$, & $0.985)$ & $61 / 64$ \\
\hline Hashmat 7 & $0.993(0.899$, & $1.000)$ & $71 / 71$ \\
\hline Bellamy 8 & $0.862 \quad(0.685$, & $0.947)$ & $25 / 29$ \\
\hline Overall $\left(I^{\wedge} 2=8326 \%, P<0.001\right)$ & $0.900(0.730$, & $0.968)$ & $272 / 300$ \\
\hline
\end{tabular}
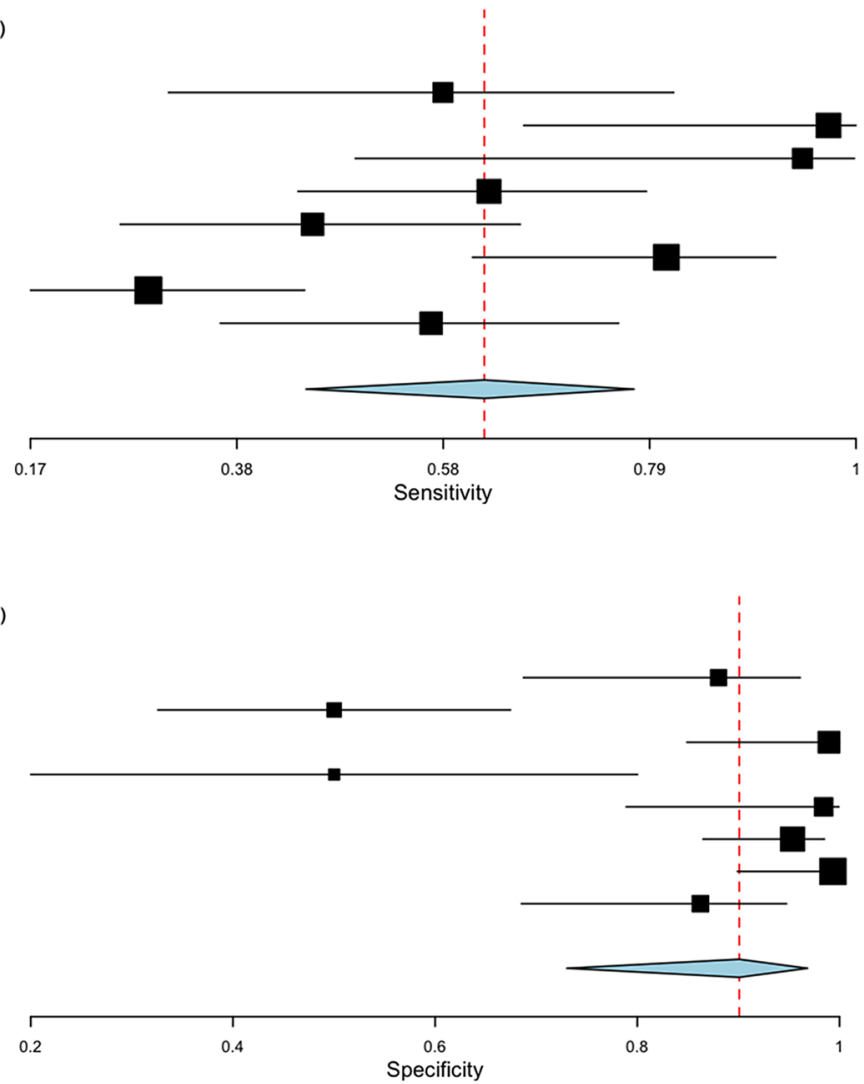

Fig. 3 Forest plot of included studies sensitivity and specificity

infarction. It is likely more specific than the V4R ECG lead and may be equally sensitive. Its use in conjunction with the right-sided precordial ECG leads, which may carry higher sensitivity could assist in early diagnosis and appropriate management of right ventricular myocardial infarction. Its diagnostic performance in the context of undifferentiated chest pain, however, remains unknown. Future research should focus on standardizing the assessment of Kussmaul's sign, which could be accomplished by demonstrating its assessment by bedside ultrasound. This would be a natural extension of the current bedside ultrasound assessment of jugular venous pressure [27]. Ultrasound assessment may result in improved sensitivity as it may identify Kussmaul's sign resulting simply in the absence of a rise in jugular venous pressure that may not be identified on physical exam. Its clinical usefulness in establishing diagnoses from undifferentiated chest pain could be explored. Regardless of future analysis, our research demonstrates that in patients with acute myocardial infarction the presence of Kussmaul's sign should be an indicator for the astute clinician to manage a patient's preload.

\section{Conclusion}

In patients with acute myocardial infarction, Kussmaul's sign is specific for acute right ventricular myocardial infarction and may serve as an important clinical sign of right ventricular dysfunction requiring preload preserving management.

Acknowledgements We thank Bruce Weaver M.Sc. Assistant Professor at the Northern Ontario School of Medicine for assistance with statistical analysis.

Funding This research received no specific grant from any funding agency, commercial or not-for-profit sectors.

\section{Compliance with ethical standards}

Conflict of interest The authors declare that they have no conflict of interest. 


\section{References}

1. Cohn JN, Guiha NH, Broder MI, Limas CJ. Right ventricular infarction: clinical and hemodynamic features. Am J Cardiol. 1974;33:209-14.

2. Hashmat A, Hashmat K, Bilal M. Right ventricular infarction in association with inferior myocardial infarction. J Med Sci. 2010;18:91-3.

3. Namana V, Gupta SS, Abbasi AA, Raheja H, Shani J, Hollander G. Right ventricular infarction. Cardiovasc Revasc Med. 2018;19:43-50.

4. Garcia-Niebla J, Perez-Riera AR, Barbosa-Barros R, Diaz-Munoz J, Daminello-Raimundo R, de Abreu LC, et al. Acute inferior myocardial infarction with right ventricular involvement and several clinical-electrocardiographic markers of poor prognosis. Ann Noninvasive Electrocardiol. 2019;24:e12592.

5. Zehender M, Kasper W, Kauder E, Schonthaler M, Geibel A, Olschewski M, et al. Right ventricular infarction as an independent predictor of prognosis after acute inferior myocardial infarction. N Engl J Med. 1993;328:981-8.

6. Richards SD. Right ventricular infarction. Aids to recognition. Postgrad Med. 1993;93:117-26.

7. Robalino BD, Whitlow PL, Underwood DA, Salcedo EE. Electrocardiographic manifestations of right ventricular infarction. Am Heart J. 1989;118:138-44.

8. Somers MP, Brady WJ, Bateman DC, Mattu A, Perron AD. Additional electrocardiographic leads in the ED chest pain patient: right ventricular and posterior leads. Am J Emerg Med. 2003;21:563-73.

9. Pandey D, Sood BR, Kaushik ML, Bhardwaj R, Sharma A. Right ventricular myocardial infarction: echocardiographic evidence among patients with inferior wall myocardial infarction. Med J Indones. 2006;15:94-9.

10. Johnson SK, Naidu RK, Ostopowicz RC, Kumar DR, Bhupathi S, Mazza JJ, et al. Adolf Kussmaul: distinguished clinician and medical pioneer. Clin Med Res. 2009;7:107-12.

11. Devine PJ, Sullenberger LE, Bellin DA, Atwood JE. Jugular venous pulse: window into the right heart. South Med J. 2007;100:1022-7.

12. Mansoor AM, Karlapudi SP. Images in clinical medicine. Kussmaul's sign. N Engl J Med. 2015;372:e3.

13. Jeffers JL, Parks LJ. Right ventricular myocardial infarction. In: StatPearls [Internet]. Treasure Island (FL): StatPearls Publishing; 2020 [cited 2020 Apr 25]. https://www.ncbi.nlm.nih.gov/books/ NBK431048/
14. Whiting PF. QUADAS-2: a revised tool for the quality assessment of diagnostic accuracy studies. Ann Intern Med. 2011;155:529.

15. Viechtbauer W. Conducting meta-analyses in $\mathrm{R}$ with the metafor package. J Stat Softw 2010;1(3) [Internet] https://www.jstatsoft. org/v036/i03

16. Baigrie RS, Haq A, Morgan CD, Rakowski H, Drobac M, McLaughlin $P$. The spectrum of right ventricular involvement in inferior wall myocardial infarction: a clinical, hemodynamic and noninvasive study. J Am Coll Cardiol. 1983;1:1396-404.

17. Bellamy GR, Rasmussen HH, Nasser FN, Wiseman JC, Cooper RA. Value of two-dimensional echocardiography, electrocardiography, and clinical signs in detecting right ventricular infarction. Am Heart J. 1986;112:304-9.

18. Cintron GB, Hernandez E, Linares E, Aranda JM. Bedside recognition, incidence and clinical course of right ventricular infarction. Am J Cardiol. 1981;47:224-7.

19. Dell'Italia LJ, Starling MR, O'Rourke RA. Physical examination for exclusion of hemodynamically important right ventricular infarction. Ann Intern Med. 1983;99:608-11.

20. Sinha N, Ahuja RC, Saran RK, Jain GC. Clinical correlates of acute right ventricular infarction in acute inferior myocardial infarction. Int J Cardiol. 1989;24:55-61.

21. Sewdarsen M, Vythilingum S, Moodley T. Incidence and bedside diagnosis of haemodynamically significant right ventricular infarction. South Afr Med J Suid-Afr Tydskr Vir Geneeskd. 1988;73:9-11.

22. Coma-Canella I, Lopez-Sendon J. Ventricular compliance in ischemic right ventricular dysfunction. Am J Cardiol. 1980;45:555-651.

23. Mittal SR, Garg S, Lalgarhia M. Jugular venous pressure and pulse wave form in the diagnosis of right ventricular infarction. Int $\mathbf{J}$ Cardiol. 1996;53:253-6.

24. Cohen SI, Kupersmith J, Aroesty J, Rowe JW. Pulsus paradoxus and Kussmaul's sign in acute pulmonary embolism. Am J Cardiol. 1973;32:271-5.

25. Cattaneo M, Muzzarelli S, Faletra F, Porretta AP, Siclari F, Gallino A. Kussmaul's sign in effusive constrictive pericarditis. Kardiovaskulare Med. 2015;18:32-3.

26. Spodick DH. Cardiac tamponade and Kussmaul's sign. Circulation. 1981;64:1078.

27. Socransky SJ, Wiss R, Robins R, Anawati A, Roy M-A, Yeung IC. Defining normal jugular venous pressure with ultrasonography. CJEM J Can Assoc Emerg Physicians Pick. 2010;12:320-4. 